\title{
An Evaluation Method for Accident Network Node Importance
}

\author{
Song Wang, Renjun Zhan and Yongzhong Ma \\ Equipment Engineering College, Engineering University of People's Armed Police, Xi'an 710086, China
}

\begin{abstract}
In order to realize the risk control of complex system, make sure its safety operation which have a number of nodes and complex associated, using implicated control of important nodes to suppression system crashes, from the perspective of accident network, based on static network node degree, node central degree from expertise, as well as risk transfer data from dynamic simulation, a node importance evaluation algorithm was presented, which used entropy weights to integrated multi-dimensional data.
\end{abstract}

KEYWORD: Accident network, node importance, evaluation, entropy.

\section{INTRODUCTION}

Complex system's fragile structure which has potentially collapse trend (WANG Song, 2011), In order to realize the risk control of complex system, make sure its safely operation which have a number of nodes and complex associated, using implicated control of important nodes to suppression system crashes. By constructing the accident system model which mapping complex systems, based on accident network structure and its dynamic behavior to achieve risk control of complex system. As Lin (LIN Wenwen, 2013) used Bayesian network to model the influences mechanism of organizational factors to crew fatigue, which can be used to study the crew fatigue levels under different network node state. Due to different nodes of accident system which have a distinction of importance, so improve the risk treatment ability of critical nodes can leverage promote the risk management capability of whole complex system?

In field of node importance research, (LI Guo, 2008) treat complex electromechanical system as a whole, and used the small-world network theory analysis method to explore the impact of the system inherent network topology characteristics and statistical characteristics to fault propagation. Chen (CHEN Jing, 2009) proposed a method to assess the importance of the node which based on nodes closeness and nodes criticality in its neighborhood. TAN (TAN Yue-jin, 2006) proposed a method to assess complex network node importance based on node shrink method, and the most important node is the node which has the largest aggregation after the aggregation of network. These methods are mostly face to static network structure, which less consider the expertise and the network dynamic behavior, however, the expertise is a reflection of the system historical data, and network dynamic behavior represent the system current data. This paper uses multi-dimensional data of static network node degree, node central degree from expertise, as well as risk transfer data from dynamic simulation to present a node importance evaluation algorithm.

\section{NODE IMPORTANCE EVALUATION METHOD}

Node Degree Data in Static Network. The complex system of mapping rules based on certain grounds of abstract nodes and edges of the accident causing the network, you can build the appropriate accident system topology model (Boccalettia S, 2006).

Definition 1. Accident system topology. Let the set of nodes $V=\left\{v_{1}, v_{2}, \ldots, v_{n}\right\}$,For the cause of all the nodes within the collection system accident. Among them $i=1,2, \ldots, n$. Cause of the number of nodes; Edge set $E=\left\{e_{i j}, i, j=1,2, \ldots, n ; i \neq j\right\}$. Collection system caused the accident because of relationships between nodes, then $G=(V, E)$ is the accident system topology.

Definition 2. The complexity of network nodes. Node degree is the number of edges directly connected to the node. Located between nodes $i$ and nodes $j(j \neq i)$ connected by edges $e_{i j}$, When exist connection between node $i$ and node $j$, Take $e_{i j}=1$, otherwise take $e_{i j}=0$. So the degree of a node $i$ can be described as 
$k_{i}=\sum_{j=1, j \neq i}^{n} e_{i j}$.

In formula (1): $\mathrm{n}$ as the total number of nodes in complex networks. In complex networks, node degree can be further subdivided into degrees and is defined as the degree of penetration refers to the number of edges entering the node, the degree is from the number of edge nodes leads. Complex network theory, the higher the degree of the nodes, the nodes show more associated therewith, relatively speaking, the greater its importance.

Because of accident the network has a hierarchical structure, so the cause of the different nodes at different hierarchy. Accident system contains three types of cause of nodes, namely the nature of causation nodes, transition nodes and cause of cause of neighboring nodes, transition nodes may contain multiple layers causation element (Buzna L, 2006).

Definition 3 Cross-layer connection. Accident network system, connected to the adjacent layer Defined as cross-layer connections. As is apparent from the nature of causation layer to the cause of the connection layer between the neighbor belongs crosslayer connections.

Cross-layer connection increases the transfer rate risk, thereby accelerating the accident. Therefore, in the definition of accident causation when the degree of the nodes must be connected to the cross-layer side is connected with the ordinary side treated differently. Is set $l$ to the number of nodes $i$ separated by layer, when $l=1$ indicating that the layer adjacent to the layer node $i$ is located, When $l=2$ indicating that the node $i$ where the layers separated by a layer, When $l=L(L=\operatorname{Max}\{l\})$ indicating the node $i$ layer of the layer where the layers $(L-1)$ apart. Accident causation networks, cross-layer connection sides must give greater weight.

Definition 4 Cause of node degree. Combined with Defined cross-layer connection, the cause of the accident can be defined system of nodes $i$ is $k^{i}$, then

$k^{i}=1 \times k_{1}^{i}+2 \times k_{2}^{i}+\ldots+l \times k_{l}^{i}+\ldots=\sum_{l=1}^{L} l \times k_{l}^{i}$.

Consider the degree of cross-layer node connection obtained better reflect the contribution node in the network and node location of the accident. By formula (2) obtained after the cause of node degree, needs to be non-dimensional processing to calculate the cause of node important degree ordering parameters $K^{i}$.

$$
K^{i}=k^{i} / \operatorname{Max}\left\{k^{i}\right\}, \quad 1 \leq i \leq n .
$$

In formula (3), the larger value of $K^{i}$, the higher the cause because of node important degree.

Center Degree Data by Expertise. Complex Systems complicated causes accidents, caused by many factors, these unstructured and semi-structured acci- dent factors structuring the deal is accident Mechanism of the basis and premise. Decision laboratory analysis (DEMATEL) can be used to determine the causal relationship between the factors is an effective tool for analyzing complex structural relationship of elements of the system. Step employs DEMATEL compute node centrality of the accident can be found in the literature (Song WANG, 2012, DU Chun, 2012).

Risk Transfer Data from Dynamic Simulation. In the literature (WANG Song, 2013), the authors using Arena software delivery process on incidents network risks entropy of the dynamic simulation analysis, consider node consume resources at the disposal of risk costs $C_{1}$, in which the cost of the use of resources for the $C_{10}$, idle resources when the cost of $C_{11}$, with each use of resources at a cost of $C_{12}$, there $C_{1}=C_{10}+C_{11}+C_{12}$.

In addition, the risk of immune system node itself also requires consumption costs, to $C_{2}$, and because of the risk of the backlog will cause the node to break the threshold value, the risk of setting the pending consumption cost $C_{3}$, then the sum of the cost of each node consumes is $C$, there is

$C=C_{1}+C_{2}+C_{3}$.

The simulation 100 , to obtain the consumption cost for each node in the process of the average value $C_{i}, C_{i}$ according to a sorting numerical size obtained results showing a large value of the cost of additional consumption required (the greater efficacy node), that is the position in the network is more important. Then sort the results obtained is dynamic and will change based on the network structure and node parameters and change.

By $C i$ dimensionless process parameters can be obtained based on the sort of dynamic transmission risk $C^{i}$

$$
C^{i}=C_{i} / \operatorname{Max}\left\{C_{i}\right\}, 1 \leq i \leq n .
$$

Finally use the entropy weight method in literature (QIU Wan-hua, 2004, CHI Baoshan, 2006 ) to integrated multi-dimensional data. Shannon defined a discrete sources produce a formula to measure the amount of information.

$$
\begin{aligned}
& H_{j}=-K \sum_{i=1}^{m} f_{i j} \ln f_{i j} . \\
& f_{i j}=\alpha_{i j} / \sum_{i=1}^{m} \alpha_{i j} . \\
& \omega_{j}=g_{j} / \sum_{j=1}^{n} g_{j}=\left(1-H_{j}\right) /\left(n-\sum_{j=1}^{n} H_{j}\right) . \\
& \sum_{j=1}^{n} \omega_{j}=1, \omega_{j} \geq 0 ; j=1,2, \ldots, n .
\end{aligned}
$$

By entropy method can be obtained node staging the final assessment of the degree of importance value is set $I_{i}$, there is 


$$
I_{i}=\omega_{1} K^{i}+\omega_{2} M^{i}+\omega_{3} C^{i} .
$$

According to the size of the final value $I_{i}$ determined can get important degree of sorting node.

\section{CASE ANALYSIS}

Based on the accident model in reference, compare the node important degree of node 2 to 14 (Where node 1 and node 15 do not consider temporarily), in accordance with entropy algorithm steps build multidimensional data, obtained entropy integrated multi-dimensional data sorted results as shown in Table 1 .

Table 1 Entropy integrated multi-dimensional data sorted results

\begin{tabular}{llllllllllllll}
\hline Nodes & 2 & 3 & 4 & 5 & 6 & 7 & 8 & 9 & 10 & 11 & 12 & 13 & 14 \\
\hline Integrated result & 0.36 & 0.47 & 0.29 & 0.33 & 0.92 & 0.25 & 0.37 & 0.31 & 0.42 & 0.66 & 0.18 & 0.85 & 0.59 \\
Sequence & 8 & 5 & 11 & 9 & 1 & 12 & 7 & 10 & 6 & 3 & 13 & 2 & 4 \\
\hline
\end{tabular}

From Table 1, node 6 has the largest importance degree, then node 13,11, 14 and 3. By the Arena simulation, the sorted result in Table 1 is the most optimized result, improve risk management capabilities of important nodes in Table 1 can maximum improve system availability.

\section{SUMMARY}

Based on accident network static network node degree, expertise node central degree, and dynamic simulation risk transfer data, presented a node importance evaluation algorithm, in which entropy weights was used to integrated the multidimensional data.

\section{ACKNOWLEDGMENT}

This study was partially supported by the National Natural Science Foundation of China with the Grant number of 71401179 .

\section{REFERENCES}

Boccalettia S, Latora, V, Moreno Y, et al. Complex networks: Structure and dynamics .Physics Reports, 2006, 424: 175308.

Buzna L, Peters K, Helbing D.Modeling the dynamics of disaster spreading in networks.Physica A, 2006(363),pp.132-140.

CHI Baoshan, LI Yong-tai, LI Jun.Technology risk assessment based on the method on entropy-weighted double base points.Science \&Technology Progress and Policy.2006(10), pp.131-133.

CHEN Jing, SUN Lin-fu.Evaluation of node importance in complex networks. Journal of Southwest Jiaotong University, 2009, 44(3),pp.426-429.

DU Chun, WANG Ying, WANG Song, GUAN Minglu.Analysis of Safety Accident Influence Factors of Complex System Based on the Integration of DEMATEL and ISM.Mathematics in Practice and Theory, 2012, 42(22), pp.143-150.

LIN Wenwen, HUANG Shuping.Bayesian Network-based analysis of effects of organizational factors on seafarer's fatigue.China Safety Science Journal, 2013, 23(6),pp.26-31.
LI Guo, GAO Jian-min, GAO Zhi-yong.Safety analysis of complex system based on small world topological model.Chinese Journal of Mechanical Engineering, 2008, 44(5),pp.86-91.

QIU Wan-hua.The modern management theory and practice.Beijing: Beijing University Press, 2004,pp.53-58.

Song WANG, Ying WANG, Chun Du.Analysis of Complex System Accident Causation Network based on DEMATELISM Method.International Journal of Digital Content Technology and its Applications, 2012, 6(17),pp.26-35.

TAN Yue-jin, WU Jun, DENG Hong-zhong.Evaluation method for node importance based on node contraction in complex networks.Systems Engineering - Theory\&Practice, 2006, 26(11), pp.79-83, 102.

WANG Song, WANG Ying.Causation analysis of complex system safety accident based on brittle structure collapse.China Safety Science Journal, 2011, 21(5),pp.138-142.

WANG Song, ZHAN Renjun, MA Yongzhong.Arena simulation of transmission of risk entropy in complex system dent causation network.China Safety Science Journal, 2013, 23(3) ,pp.27-32.

WANG Song.Model construction analysis of complex system accident causation network.China Safety Science Journal, 2013, 23(2), pp. 109-116. 